\title{
The question-behavior effect from an action control perspective
}

\author{
Peter M. Gollwitzer*, Gabriele Oettingen* \\ Psychology Department, New York University, 6 Washington Place, New York, NY 10003, USA
}

\begin{abstract}
The question-behavior effect is discussed by taking an action control perspective. With respect to the determinants of the effect, it is asked whether its strength is dependent on the features of the behaviors at issue (e.g., difficulty), how the question targets the critical behaviors (e.g., in terms of attitudes or intentions, frequency or intensity), and in which context it is asked. With respect to the psychological processes, it is questioned whether the activation of positive implicit attitudes underlies the effect. Finally, to counter unwanted question-behavior effects on risky behaviors, an intervention treatment geared at blocking such effects is suggested that is based on the self-regulation of goal setting (by mental contrasting; Oettingen, 2000) and goal implementation (by forming if-then plans; Gollwitzer, 1999).
\end{abstract}

The psychology of action (Gollwitzer \& Bargh, 1996; Oettingen \& Gollwitzer, 2001) has developed conceptual tools that allow for the analysis of questions such as: When is action control easy or difficult, failure-ridden or successprone, conscious and effortful or automatic and efficient? Research using these conceptual tools sheds light on how action control is guided by people's motives, their wishes and attitudes, their various kinds of expectations, goals, and plans as well as their evaluative thoughts with respect to achieved outcomes. In the present commentary, we will use the research perspective of the psychology of action to reflect on the question-behavior effect as discussed by Fitzsimons and Moore (2008).

Fitzsimons and Moore caution to ask adolescents about risky behaviors (e.g., drugs, alcohol, dangerous sports, unsafe sex, smoking), because this might increase the frequency of engaging in exactly these risky behaviors. Shall parents therefore stay away from asking their children about risky behaviors? And shall researchers and educators stop conducting individual and population screening? On a first glance, it always seems to be beneficial for parents, educators, health professionals and people who design public policies to learn about the extent of adolescents' engagement in various kinds of risky behaviors, as such information yields important benefits for the

\footnotetext{
* Corresponding authors.

E-mail addresses: Peter.Gollwitzer@nyu.edu (P.M. Gollwitzer), Gabriele.Oettingen@nyu.edu (G. Oettingen).
}

individual, the care taker, and the society at large (e.g., individuals can avoid tempting situations, caretakers can encourage alternative engagements, researchers and policy makers can corroborate in developing targeted intervention programs for prevention and cure). But Fitzsimons and Moore raise an intriguing counter-argument stating that these benefits may be offset by the question-behavior effect: People who are asked about a particular behavior show an increased probability of carrying out this very behavior.

In our opinion, the question of costs and benefits of asking people about their risky behaviors is a question that the relevant decision makers (e.g., parents, politicians, health professionals) have to answer for themselves. The scientists, on the other hand, have to deliver the necessary factual information. More specifically, scientists are to provide to decision makers factual information on the strength of the question-behavior effect, its determinants and processes, and how it can be prevented and attenuated. In the following, we will address these issues one by one.

\section{Strength of the question-behavior effect}

With respect to the first question (how strong is the effect?), a sophisticated meta-analysis is needed that considers all of the individual studies on the question-behavior effect that have been conducted so far. With a sophisticated analysis we mean that differential $d$ s are computed for different types of samples (e.g., children, adolescents, adults; students, professionals; men, women), different types of behaviors 
(e.g., consumer, risky, communication), and behavior in different life domains (e.g., interpersonal, achievement, health). Regarding the Fitzsimons and Moore paper, it would be important to know whether risky behaviors show significantly different $d s$ for the question-behavior effect than non-risky behaviors, and whether the behavior-question effects for these risky behaviors are of a small, medium, or large magnitude. It is vital to know for computing reliable costbenefit analyses (of whether one should ask adolescents about certain risky behaviors) how strong the question-behavior effects actually are for the risky behaviors at issue.

\section{Determinants of the question-behavior effect}

Let us now turn to the determinants of the question-behavior effect. First, an important determinant of the strength of the effect may be features of the risky behavior itself. For example, it is possible that only easy to implement behaviors are vulnerable to the effect (e.g., accepting an offered drug) while more difficult to implement behaviors are protected from it (e.g., trying to buy drugs). The strength of the question-behavior effect might also depend on whether the critical behavior is linked to norms embraced by the majority of a society (sun bathing, alcohol consumption) or to norms embraced only by a sub-culture (drug use, tattooing).

Second, the question-behavior effect may also be determined by whether the question targets the critical behavior directly or indirectly. Questions targeting the behavior indirectly may focus on the psychological concepts related to this behavior such as relevant attitudes, expectations, and goals (intentions), as well as plans to carry out the behavior. If attitudes are targeted, what dimension of attitude is inquired about (e.g., ambivalence)? If expectations are targeted, how are they conceptualized (in terms of efficacy, outcome expectations, or general expectations)? Are the certainties of holding these expectancies asked for as well? If goals (intentions) are assessed, is it the strength of the commitment or the quantity and quality of the standard? And if the set standard is asked for, does it pertain to a specific performance outcome or to merely doing one's best? If a plan is asked about, is it an outcome versus process simulation, or an ifthen statement (i.e., an implementation intention that specifies what goal-directed behavior one wants to perform if a certain critical situation will be encountered)?

Third, already the mere framing of the question may weaken or strengthen the question-behavior effect. Fitzsimons and Moore mention temporal focus (future versus past), valence focus (positively versus negatively), and means focus (approach versus avoidance) as well as the anchoring of the answer scale ( 7 times a week versus 8 times a week). Whether the question asks for frequency versus intensity of the behavior might also play a role.

Fourth, the context as well as the physical and temporal location in which the question is asked might be a determinant of the question-behavior effect. For example, does the question appear in the context of other questions also addressing risky behavior or among questions addressing comparatively neutral behaviors? What reference points do the instructions use? For example, do the instructions implicitly convey the assumption that similar others are engaging in risky behavior too, that engaging in these risky behaviors is known or even accepted by the adult world, or that the risky behavior is a means to assert oneself against authority? Further, in what location do the adolescents answer the question? Are they providing their answer outside the critical context long before they are faced with the opportunity to act in a risky way (e.g., in school) or while they are already facing the decision to act on the risky behavior (e.g., on the internet or in public places such as parties or cafes)?

\section{Psychological processes underlying the question-behavior effect}

Fitzsimons and Moore propose that asking adolescents about their risky behaviors activates their implicit positive attitudes toward these behaviors which in turn facilitate carrying out these behaviors. Such a process hypothesis assumes a) that adolescents have implicit positive attitudes with respect to risky behaviors, and b) that it is the activation of these implicit positive attitudes what leads to risky behavior. Are these assumptions justified?

What is the empirical basis of the assumption that adolescents hold implicit positive attitudes towards risky behaviors? Are there studies systematically assessing adolescents' implicit attitudes towards risky behaviors by using various implicit measures of attitudes (e.g., the Implicit Association Test, IAT; the auto-evaluation test)? Do studies using these procedures indeed detect implicit positive attitudes towards risky behavior in adolescents? How strong are these positive attitudes, and how stable are they over time?

With respect to the second assumption (the activation of implicit positive attitudes leads to risky behavior), we wondered whether a positive implicit attitude activated in the questioning situation stays in a state of activation until the opportunity to act is finally coming along. Such an enduring heightened state of activation seems rather unlikely as the activation of mental constructs is commonly known to decay over time. Thus the activation of an implicit positive attitude may produce questionbehavior effects only when the adolescents answer the question in the same context in which they also have an opportunity to perform the risky behavior (see the moderating role of context as pointed out above).

Therefore, we suggest that questioning adolescents about risky behaviors may not only affect the state of activation of the implicit attitude but also increase the level of positivity of the implicit attitude. In order to test these competing (or complementing) process hypotheses, studies that allow computing mediation analyses would need to be conducted. In such studies, adolescents are first asked about risky behaviors, and then classic procedures of assessing the activation status versus the positivity of their implicit attitudes towards these behaviors are employed. Finally, participants are given the chance to enact the risky behaviors, and this opportunity would be provided either immediately or delayed. Statistical analyses that support 
the positivity rather than the activation hypothesis would need to show that questioning adolescents about risky behavior increases the positivity of their attitudes towards the risky behavior, and that this increase in positivity accounts for the risky behavior, immediately as well as delayed. It is important to note that all of the psychological concepts discussed above (e.g., attitudes, expectations, intentions, goals, plans) do qualify as potential alternative mediators, and a sophisticated analysis of the psychological processes underlying the questionbehavior effect would have to consider these alternative mediators (alternative to implicit attitudes) in comparative mediation analyses.

\section{Prevention and attenuation of the question-behavior effect}

Fitzsimons and Moore rightfully argue that given the question-behavior effect on risky behaviors, adolescents who participate in individual or population screening should be given an intervention treatment geared at blocking this effect. They note that such interventions should respect adolescents' need for autonomy and foster their development as independent individuals. Therefore, interventions that provide adolescents with self-regulation tools that can be administered in an autonomous and self-paced way should be particularly helpful.

To date, self-regulation interventions typically tell people how to strive for a priori defined goals to resist risky behaviors. In such interventions, participants are not encouraged to learn strategies to be applied to their own idiosyncratic risks. Rather, they are asked to engage in certain goal-directed thoughts, feelings, and actions targeted specifically at resisting a given pre-defined risky behavior. However, in everyday life, adolescents commonly need to resist a multitude of different risky behaviors (e.g., alcohol, drugs, smoking). Therefore, adolescents should benefit from metacognitive knowledge about strategies that are content free as they relate to prioritizing and planning out goal pursuit in general. Indeed, there is a recent study showing that the selfregulation strategy of mentally contrasting an envisioned positive future with obstacles of present reality (Oettingen, 2000; Oettingen, Pak, \& Schnetter; 2001) can be successfully taught and used as a metacognitive strategy to improve selfdiscipline (Oettingen, Mayer, \& Brinkmann, submitted for publication (b)). Personnel managers who completed a daily mental contrasting exercise for 2 weeks about self-identified worrisome problems reported better time management, more ease of decision-making, and more effective project completion, compared to those who performed a control exercise (i.e., had to think of but did not mentally contrast the desired future outcomes).

In a follow-up study (Oettingen, Barry, Guttenberg, \& Gollwitzer, submitted for publication (a)), we trained college students to use mental contrasting (MC; summary by Oettingen \& Thorpe, 2006) and combine it with implementation intentions (II; summaries by Gollwitzer, 1993, 1999) as a metacognitive strategy. That is, we first taught this MCII technique and then showed participants how to apply the technique to their own everyday concerns. Because we taught participants how to use a metacognitive strategy to be applied to a broad spectrum of problems, we measured improvements in relatively general personal attributes (self-discipline, selfesteem) rather than performance on specific tasks or goals. As a metacognitive strategy for the self-regulation of goal pursuit, we examined the combination of MC and II, because the combination of the two should be particularly effective for goal attainment. Specifically, in order to be effective, plans in the form of implementation intentions require that strong goal commitments are in place (Gollwitzer \& Sheeran, 2006; Sheeran, Webb, \& Gollwitzer, 2005, Study 1), and mental contrasting (prior to forming implementation intentions) creates such strong commitments. Additionally, mental contrasting aids in the recognition of obstacles that hinder goal striving. These same obstacles can then be addressed with "ifthen" plans by specifying critical situations in the ifcomponent that are linked to instrumental goal-directed responses in the then-component. Moreover, mental contrasting increases a person's readiness to make "if-then" plans (Oettingen et al., 2001); accordingly, an intervention such as MCII which explicitly suggests forming if-then plans after mental contrasting can capitalize on this effect.

Assuming that MCII improves self-regulation of goal pursuit, in our college student study we looked at changes in self-discipline and even self-esteem, and indeed observed improvements on both variables already after a one week of using MCII. In further MCII-intervention studies, the beneficial effects of the intervention were also observed with respect to increasing study behavior in high school students (over a period of three months), with respect to increasing healthy eating in adults (over a period of 2 years), and with respect to exercising in healthy adults and in chronic back pain patients (over periods of 4 and 3 months, respectively).

In sum, MCII interventions increase long-term selfdiscipline regarding studying and life management in students as well as health behaviors in adults by providing the selfregulation tools of $\mathrm{MC}$ and II that people can use by themselves with regard to their idiosyncratic problems and concerns. Therefore, MCII interventions may be a welcome tool for adolescents to prevent and stop engaging in risk behavior. Specifically, MCII interventions may be used to commit to and implement three types of goals: prevention goals geared at avoiding situations that are conducive to risk behaviors (e.g., stock hard drinks), interruption goals geared at stopping risk behavior in its course (e.g., leave a drinking party, use a condom), and approach alternative goals geared at building fascinating alternatives to risky behavior (e.g., join a soccer club, engage in flight simulator games).

\section{Conclusion}

Fitzsimons and Moore have pointed to a potentially critical disadvantage of the common and beneficial practice of asking adolescents about risk behaviors (e.g., drugs, alcohol, smoking). The authors argue that the question-behavior effect may actually worsen the very same behavior that educators and policy 
makers want to attenuate by consulting the upcoming generation. We caution against prematurely abolishing questionnaire techniques that ask adolescents to report on risk behavior. Before educators and policy makers can make reasonable decisions about costs and benefits of questioning adolescents regarding risk behavior, scientists need to establish the strength of the question-behavior effect, its determinants and processes, and whether and how it can best be attenuated.

\section{References}

Fitzsimons, G. J., \& Moore, S. G. (2008). Should we ask our children about sex, drugs and rock \& roll? Potentially harmful effects of asking questions about risky behaviors. Journal of Consumer Psychology, 18.

Gollwitzer, P. M. (1993). Goal achievement: The role of intentions. European Review of Social Psychology, 4, 141-185.

Gollwitzer, P. M. (1999). Implementation intentions: Strong effects of simple plans. American Psychologist, 54, 493-503.

Gollwitzer, P. M. \& Bargh, J. A. (Eds.). (1996). The psychology of action: Linking cognition and motivation to behavior New York: Guilford Press.
Gollwitzer, P. M., \& Sheeran, P. (2006). Implementation intentions and goal achievement: A meta-analysis of effects and processes. Advances in Experimental Social Psychology, 38, 69-119.

Oettingen, G. (2000). Expectancy effects on behavior depend on selfregulatory thought. Social Cognition, 18, 101-129.

Oettingen, G., Barry, H., Guttenberg, K. B., \& Gollwitzer, P. M. (submitted for publication). Improving self-discipline and self-esteem: A mental contrasting with implementation intentions intervention.

Oettingen, G., \& Gollwitzer, P. M. (2001). Goal setting and goal striving In A. Tesser \& N. Schwarz (Eds.), The Blackwell Handbook of Social Psychology (pp. 329-347). Oxford: Blackwell.

Oettingen, G., Mayer, D., \& Brinkmann. (submitted for publication). Mental contrasting of future and reality: Managing the demands of everyday life.

Oettingen, G., Pak, H., \& Schnetter, K. (2001). Self-regulation of goal setting: Turning free fantasies about the future into binding goals. Journal of Personality and Social Psychology, 80, 736-753.

Oettingen, G., \& Thorpe, J. S. (2006). Fantasy realization and the bridging of time. In L. A. Sanna \& E. C. Chang (Eds.), Judgments over time: The interplay of thoughts, feelings, and behaviors (pp. 120-142). Oxford: Oxford University Press.

Sheeran, P., Webb, T. L., \& Gollwitzer, P. M. (2005). The interplay between goal intentions and implementation intentions. Personality and Social Psychology Bulletin, 31, 87-98. 